\title{
FATORES DE RISCO PARA INFARTO AGUDO DO MIOCÁRDIO EM PACIENTES IDOSOS CADASTRADOS NO PROGRAMA HIPERDIA
}

\author{
Raul Henrique Oliveira Pinheiro', Maria Cristina Umpiérrez Vieira², Evani Marques Pereira², \\ Maria Emilia Marcondes Barbosa ${ }^{2}$
}

\begin{abstract}
RESUMO: Estudo quantitativo teve por objetivo identificar a prevalência dos fatores de risco relacionados ao Infarto Agudo do Miocárdio em 258 pacientes idosos cadastrados no programa Hiperdia em três unidades de saúde de Guarapuava - Paraná. Os dados foram coletados em domicílio no período de junho de 2010 a julho de 2011, utilizando questionário semiestruturado e analisados com auxílio de estatística descritiva. Os resultados mostraram que $72,49 \%$ eram mulheres com média de idade de 72,2 anos. Evidenciaram-se como fatores de risco o sedentarismo (55,81\%), hereditariedade (53,87\%), ingesta hipercalórica $(27,51 \%)$, tabagismo $(25,58 \%)$, obesidade $(24,8 \%)$ e alcoolismo $(8,52 \%)$. Considerando serem, em sua maioria, fatores de risco modificáveis, salienta-se a importância de estratégias que reorientem e direcionem as ações de controle para além da medicalização, contemplando as particularidades dos idosos nas ações de educação à saúde.

DESCRITORES: Idoso; Fatores de risco; Infarto do miocárdio; Enfermagem.
\end{abstract}

\section{RISK FACTORS FOR ACUTE MYOCRADIAL INFARCTION IN ELDERLY PATIENTS REGISTERED IN THE HIPERDIA PROGRAM}

\begin{abstract}
This qualitative study aimed to identify the prevalence of the risk factors related to Acute Myocardial Infarction in 258 elderly patients registered in the Hyperdia program in three health centers in Guaparava, in the state of Parana. The data was collected in the patients' homes in the period June 2010 to July 2011, using a semi-structured questionnaire, and was analyzed with the help of descriptive statistics. The results showed that $72.49 \%$ were women, with an average age of 72.2 years. The following were evidenced as risk factors: sedentarism (55.81\%), heredity (53.87\%), hypercaloric intake (27.51\%), smoking (25.58\%), obesity (24.8\%) and alcoholism $(8.52 \%)$. Considering that the majority of these are modifiable risk factors, the importance of strategies which re-orient and direct control actions beyond medicalization stands out, taking into account the elderly people's particular characteristics in health education actions. DESCRIPTORS: Aged; Risk factors; Myocardial infarction; Nursing.

\section{FACTORES DE RIESGO PARA INFARTO AGUDO DEL MIOCARDIO EN PACIENTES ANCIANOS CADASTRADOS EN EL PROGRAMA HIPERDIA}

RESUMEN: Estudio cuantitativo que tuvo el objetivo de identificar la prevalencia de los factores de riesgo acerca del Infarto Agudo del Miocardio en 258 pacientes ancianos cadastrados en el programa Hiperdia en tres unidades de salud de Guarapuava - Paraná. Los datos fueron recogidos en domicilio en el período de junio de 2010 a julio de 2011, utilizando cuestionario semiestructurado y analizados con la ayuda de estadística descriptiva. Los resultados muetran que 72,49\% eran mujeres con media de edad de 72,2 años. Los factores de riesgo evidenciados fueron el sedentarismo (55,81\%), hereditariedad (53,87\%), ingesta hipercalórica (27,51\%), tabaquismo (25,58\%), obesidad (24,8\%) y alcoholismo (8,52\%). Ya que son considerados, en su mayoría, factores de riesgo modificables, se ressalta la importancia de estrategias para reorientar y direccionar las acciones de control para además de la medicalización, contemplando las particularidades de los ancianos en las acciones de educación a la salud. DESCRIPTORES: Anciano; Factores de riesgo; Infarto del miocardio; Enfermería.

\footnotetext{
${ }^{1}$ Acadêmico de Enfermagem da Universidade Estadual do Centro Oeste - UNICENTRO, Campi CEDETEG - Guarapuava, PR. ${ }^{2}$ Enfermeira. Professora do Curso de Graduação em Enfermagem da UNICENTRO - CEDETEG. Membro do Grupo de Pesquisa Cuide-Vita.
} 


\section{INTRODUÇÃO}

A Organização Mundial de Saúde (OMS) mostra que $60 \%$ do ônus decorrente de doenças no mundo é determinado por problemas crônicos, principalmente doenças cardiovasculares. A OMS estima também que em 2020 esse ônus será de $80 \%$ nos países em desenvolvimento $^{(1)}$. No Brasil as doenças do aparelho circulatório foram a principal causa de óbitos em 2005, representado 32,2\% (283.927 óbitos) causados por esses agravos $^{(2-3)}$. Dentre as doenças do aparelho circulatório, os distúrbios isquêmicos do coração destacam-se como as principais causas de óbito, em especial o Infarto Agudo do Miocárdio (IAM). Em 2005, a taxa de mortalidade especifica para os distúrbios isquêmicos foi de 46,01\%, acima de países como Espanha, França, Argentina, Japão, Cuba e Estados Unidos ${ }^{(4-5)}$.

O IAM tem maior taxa de mortalidade em regiões desenvolvidas do Brasil e do mundo; para cada cinco ou sete casos desses distúrbios um evolui para óbito ${ }^{(6)}$. Os indicadores tendem a crescer com o passar do tempo, impulsionadas pelo crescimento e envelhecimento da população, também relacionados a hábitos de vida prejudiciais à saúde conhecidos, como fatores de risco.

Considerando que o IAM é a principal causa de morte entre as doenças cardiovascular em idosos, é fundamental abordar os fatores de risco no contexto dos condicionantes socioeconômicos, culturais e ambientais a fim de propor ações de prevenção e controle, contextualizadas na realidade, conforme os recursos disponíveis ${ }^{(3)}$. As patologias associadas a maior risco de IAM são a Hipertensão Arterial Sistêmica (HAS) e o Diabetes Mellitus (DM). O Ministério da Saúde (MS) avaliou o impacto dessas doenças nos agravos cardiovasculares usando como referência a Portaria n. 493 de 13 de março de 2006. A referida portaria aprova a relação de indicadores da atenção básica, estima-se que a taxa de portadores de DM e HAS na população acima de 40 anos seja de $11 \%$ e $35 \%$, respectivamente. No Brasil, entre a população idosa acima de 60 anos, estima-se que mais de 9 milhões sejam portadores de HAS e DM, $80 \%$ deles são tratados na rede publica de saúde. No Estado do Paraná a quantidade de idosos portadores de HAS e DM é de 539.000 idosos $^{(7)}$.

A fim de prevenir agravos e promover a qualidade de vida de pessoas portadoras de HAS e DM, o MS estabeleceu o Programa Hiperdia como estratégia de tratamento e acompanhamento ${ }^{(2)}$. No contexto deste estudo as ações do Programa Hiperdia estavam principalmente centradas na distribuição de medicamentos.
Ações conjuntas da equipe interdisciplinar, focadas na promoção da saúde e prevenção de agravos por meio da identificação e controle dos fatores de risco para eventos cardiovasculares eram tímidas ou inexistentes.

Estas informações geraram o interesse de investigar os fatores de riscos para IAM que estão expostos os idosos cadastrados no Programa Hiperdia. Esta iniciativa vai ao encontro da necessidade de desenvolver pesquisas como estratégia para contribuir com iniciativas que busquem melhorar o perfil epidemiológico em determinada população. A medicalização, desarticulada de outras ações de saúde, mostrou-se insuficiente para o controle dos eventos cardiovasculares. No contexto do Programa é relevante conhecer os fatores de risco que a população está exposta para obter uma base de dados, e assim monitorar estes fatores. A proposta do MS é que sejam realizados inquéritos, principalmente a nível local, aplicado a grupos vulneráveis e cujos resultados contribuam para reorientar as políticas específicas de redução de fatores de riscos nesses grupos $^{(8)}$.

Desta forma, o objetivo deste estudo foi identificar a prevalência de fatores de risco para o IAM na população idosa cadastrada no Programa Hiperdia na cidade de Guarapuava - Paraná.

\section{MÉTODO}

Estudo transversal de base populacional, realizado por meio de visita domiciliar. A coleta de dados se deu no período de junho de 2010 a julho de 2011, em três Unidades Básicas de Saúde - e respectivas áreas de abrangência - cuja população estudada totalizou 258 idosos. Os endereços dos participantes foram obtidos mediante análise dos prontuários dos pacientes cadastrados no Programa Hiperdia.

Os critérios de inclusão foram ter idade superior a 60 anos e ser cadastrados no Programa Hiperdia. Os critérios de exclusão foram ser portador de condições clínicas que impedissem a realização da coleta de dados, impossibilidade de localização do idoso no domicilio após três tentativas, e mudança de endereço durante o estudo.

A coleta de dados foi realizada no domicilio dos participantes, utilizando-se um questionário com questões fechadas, em formato check-list, e questões abertas. Os dados sociodemográficos foram obtidos por meio das questões fechadas, no entanto, as questões abertas forneceram informações específicas, como hábitos de vida e histórico de doenças. O preenchimento 
do instrumento foi realizado pelos pesquisadores. As variáveis independentes foram: sexo, idade, escolaridade, situação conjugal, raça e renda em salários. Para identificar os fatores de risco cardiovasculares, as variáveis utilizadas foram alcoolismo, tabagismo, obesidade, ingesta hipercalórica, sedentarismo, hereditariedade e condições crônicas. A análise de dados foi realizada após dupla digitação em um banco de dados elaborado para este estudo com auxílio do programa Estatística 7.0 que permite calcular as medidas estatísticas. Este estudo teve aprovação do Comitê de Ética em Pesquisa da Universidade Estadual do Centro-Oeste, mediante parecer número 366/2010. Todos os participantes formalizaram a participação assinando o Termo de Consentimento Livre e Esclarecido e foram observados os preceitos de ética em pesquisa.

\section{RESULTADOS}

Dos 290 idosos cadastrados no Programa Hiperdia, respeitando os critérios de inclusão e exclusão, participaram da pesquisa 258 idosos $(88,96 \%)$, sendo $27,51 \%$ homens e $72,49 \%$ mulheres. A média de idade foi de 72,2 anos com um desvio padrão de 8,1 e a mediana de 71 anos. Os idosos foram classificados em três faixas etárias: 60 a 69 anos (43,54\%), 70 a 79 anos (36,35\%), e 80 anos ou mais (20\%). A maioria era casada, de cor branca, renda salarial mensal de no máximo um salário mínimo e escolaridade de no máximo quatro anos.

Com relação aos fatores de risco cardiovascular, houve o predomínio de sedentarismo $(55,81 \%)$, here- ditariedade $(53,87 \%)$ ingesta hipercalórica $(27,51 \%)$, tabagismo (25,58\%), obesidade $(24,80 \%)$ e alcoolismo $(8,52 \%)$, como mostra a figura 1 , em ordem decrescente e com os respectivos percentuais.

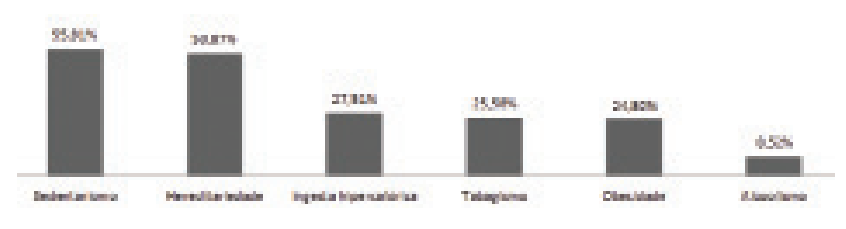

Figura 1 - Prevalência de fatores de risco para infarto agudo do miocárdio em idosos (n=258). Guarapuava, 2011

Ao relacionar os fatores de risco com as faixas etárias, identificou-se que alcoolismo, hereditariedade e sedentarismo aumentaram conforme a idade avançava, enquanto que obesidade, ingesta hipercalórica e tabagismo apresentaram queda com o avanço da idade, principalmente após os 80 anos, sugerindo que estes últimos três fatores estão associados a maiores taxas de mortalidade. A tabela 1 mostra os respectivos porcentuais.

Em relação ao sexo, a ingesta hipercalórica e o alcoolismo foram prevalentes nos homens. $\mathrm{O}$ tabagismo e a obesidade prevaleceram entre as mulheres e os fatores hereditariedade e sedentarismo apresentaram-se em taxas semelhantes em ambos os sexos. Entre os participantes do estudo, o distúrbio crônico prevalente foi a HAS.

Tabela 1- Fatores de risco para infarto agudo do miocárdio em idosos conforme a faixa etária (n=258). Guarapuava, 2011

\begin{tabular}{|c|c|c|c|c|c|c|c|c|c|}
\hline \multirow{2}{*}{\multicolumn{2}{|c|}{ Fatores de Risco }} & \multicolumn{6}{|c|}{ Faixa etária (em anos) } & \multirow{2}{*}{\multicolumn{2}{|c|}{$\begin{array}{c}\text { Total } \\
(\mathrm{n}=258)\end{array}$}} \\
\hline & & \multicolumn{2}{|c|}{$60-69(n=113)$} & \multicolumn{2}{|c|}{$70-79(n=92)$} & \multicolumn{2}{|c|}{80 ou mais $(n=53)$} & & \\
\hline & & $\mathrm{n}$ & $\%$ & $\mathrm{n}$ & $\%$ & $\mathrm{n}$ & $\%$ & $\mathrm{n}$ & $\%$ \\
\hline \multirow{2}{*}{ Alcoolismo } & $\operatorname{sim}$ & 6 & 5,30 & 8 & 8,69 & 8 & 15,09 & 22 & 8,52 \\
\hline & não & 107 & 94,70 & 84 & 91,31 & 45 & 84,91 & 236 & 91,48 \\
\hline \multirow{2}{*}{ Hereditariedade } & $\operatorname{sim}$ & 50 & 44,24 & 55 & 59,78 & 34 & 64,15 & 139 & 53,87 \\
\hline & não & 63 & 55,76 & 37 & 40,22 & 19 & 35,85 & 119 & 46,13 \\
\hline \multirow{2}{*}{ Sedentarismo } & $\operatorname{sim}$ & 60 & 53,09 & 51 & 55,43 & 33 & 62,26 & 144 & 55,81 \\
\hline & não & 52 & 46,91 & 41 & 44,57 & 20 & 37,74 & 114 & 44,19 \\
\hline \multirow{2}{*}{ Obesidade } & $\operatorname{sim}$ & 38 & 33,62 & 22 & 23,91 & 4 & 7,54 & 64 & 24,80 \\
\hline & não & 75 & 66,38 & 70 & 76,09 & 49 & 92,46 & 194 & 75,20 \\
\hline Ingesta & $\operatorname{sim}$ & 38 & 33,62 & 28 & 30,43 & 5 & 9,43 & 71 & 27,51 \\
\hline Hipercalórica & não & 75 & 66,38 & 64 & 69,57 & 48 & 90,57 & 187 & 72,49 \\
\hline \multirow{2}{*}{ Tabagismo } & $\operatorname{sim}$ & 33 & 29,20 & 22 & 23,91 & 11 & 20,75 & 66 & 25,58 \\
\hline & não & 80 & 70,80 & 70 & 76,09 & 42 & 79,25 & 192 & 74,42 \\
\hline
\end{tabular}

Cogitare Enferm. 2013 Jan/Mar; 18(1):78-83 


\section{DISCUSSÃO}

Diversos estudos comprovam o predomínio do sexo feminino ${ }^{(9-10)}$ entre participantes cadastrados no Programa Hiperdia, reflexo de que no Brasil a população feminina idosa é maior. Conforme dados revelados pelo Instituto Brasileiro de Geografia e Estatística, nas faixas etárias avançadas, o número de mulheres é significativamente maior que a quantidade de homens ${ }^{(7)}$.

O maior percentual de idosos foi na faixa etária até 69 anos, porém considerou-se que o menor número de idosos longevos está relacionado às taxas de mortalidade maiores à medida que avança a idade. Este dado vem ao encontro das evidências do inquérito nacional domiciliar, realizado em 16 capitais brasileiras ${ }^{(10)}$, no qual a faixa etária prevalente foi de 60 a 69 anos, representando $55,44 \%$ da população.

O IAM pode ser considerado um distúrbio de idosos, pois maioria das mortes ocorre acima dos 65 anos $^{(11)}$. Porém, há que considerar que os riscos para o desenvolvimento de doenças cardiovasculares aumentam consoante a idade avança, não porque a pessoa envelhece, mas porque quanto maior o tempo de exposição aos fatores de risco, maior a probabilidade de acontecer um evento cardiovascular adverso ${ }^{(12)}$.

Evidenciou-se que o alcoolismo aumentou com o avanço da idade. Este fato pode estar relacionado à atitude permissiva de consumo de álcool em décadas passadas, quando o álcool não era considerado potencialmente perigoso, e seu uso era incentivado para alterar o humor, melhorar o apetite ou induzir o sono ${ }^{(13)}$. A mudança comportamental, em relação ao uso abusivo de álcool e sua interrupção, exige acompanhamento profissional, familiar e de grupos de apoio de maneira contínua, trabalho este que desafia os profissionais de enfermagem a desenvolver ações de educação para a saúde em todas as faixas etárias.

O hábito de fumar foi constatado em $25,58 \%$ dos idosos entrevistados, e houve prevalência entre as mulheres. Em um estudo realizado na cidade de Ribeirão Preto, o número de mulheres tabagistas foi expressivamente maior em relação aos homens, e a população investigada mostrou dificuldade para identificar o hábito de fumar como fator de risco importante para ocorrência do IAM ${ }^{(10)}$. Estes dados são preocupantes, pois a probabilidade de sofrer um IAM é, em média, quatro vezes maior entre mulheres fumantes ${ }^{(14)}$. A interrupção deste hábito requer ações específicas interdisciplinares e redes de apoio, pois somente a divulgação de informações a respeito dos efeitos negativos do cigarro não têm se mostrado suficiente para o controle do uso tabaco, cabendo ao profissional enfermeiro integrar-se aos esforços interdisciplinares para o controle do tabagismo.

O único fator de risco cardiovascular não modificável avaliado nesta pesquisa - a hereditariedade apresentou taxa de prevalência de 53,87\%. A história familiar de doenças cardiovasculares em parentes de primeiro grau aumenta a chance de sofrer IAM em até duas vezes ${ }^{(15)}$. Esta realidade exige do enfermeiro habilidade e competência para identificar fatores de risco e para a elaboração de estratégias, adequadas ao idoso, com vistas a favorecer a interrupção precoce de hábitos modificáveis e contribuintes à ocorrência de IAM.

O sedentarismo foi o fator de risco que apresentou a maior taxa de prevalência $(55,81 \%)$. A inatividade física é um dos principais e mais frequentes fatores de risco cardiovascular. É um estilo de vida considerado sedentário leva a um risco 1,9 maior para o IAM quando comparado a uma pessoa com atividade física programada ${ }^{(16)}$. As altas taxas de inatividade física nos idosos brasileiros podem estar relacionadas à falta de orientação por parte dos profissionais de saúde, bem como de lugares apropriados para a realização destas atividades. A atividade física, além de diminuir o risco cardiovascular, promove maior interação social entre idosos e a independência nas atividades da vida diária ${ }^{(10)}$. $\mathrm{O}$ incentivo à realização de exercícios físicos contribui para interferir não apenas no fator de risco sedentarismo, mas nos fatores obesidade e depressão.

Neste estudo, considerou-se, uma alimentação inadequada aquela rica em gorduras e pobre em verduras e frutas. A taxa de ingesta hipercalórica foi de (27,51\%). A questão alimentar é de grande importância na ocorrência de eventos cardiovasculares, as intervenções nutricionais envolvem atenção aos fatores de risco, como sedentarismo, obesidade e alimentação inadequada. $\mathrm{O}$ aumento da adesão a um regime alimentar de boa qualidade pode diminuir as taxas de mortalidade da população com agravo cardiovascular em até $18 \%{ }^{(17)}$. Para tanto, cabe ao profissional enfermeiro conhecer e identificar a alimentação adequada, promovendo informações necessárias para a criação deste hábito de saúde.

A obesidade esteve estreitamente relacionada com o fator de risco na ingesta hipercalórica e aos hábitos de saúde, como o uso excessivo de sal nos alimentos, alimentação rica em gordura animal e pouca ingesta de verduras e legumes. Quanto maior o Índice de Massa Corpórea, maior é a taxa de obesidade e maior o risco para a ocorrência de um agravo cardiovascular ${ }^{(18)}$. Embora nos últimos anos os homens tenham tido grande 
aumento nas taxas relacionadas à obesidade, esta ainda é um fator de risco cardiovascular que está mais relacionada ao sexo feminino. O menor acometimento do fator de risco obesidade ao sexo masculino pode estar relacionado à maior prática de atividade física por homens e aos fatores de mudanças hormonais nas mulheres ${ }^{(10)}$.

O fato de que para alguns fatores de risco as taxas diminuíram com o avanço da idade não evidencia que as ações realizadas em saúde são efetivas ou que a população tenha abandonado estes hábitos. Altas taxas de mortalidade ocasionadas por esses fatores de risco não permitem que a população chegue até idades mais avançadas ${ }^{(19)}$.

Neste estudo a HAS esteve estreitamente relacionada a todos os fatores de risco, em todas as faixas etárias e em ambos os sexos, sendo que também foi a doença crônica de maior incidência. Sabe-se que este agravo poderia ser controlado ao ser diagnosticado e tratado precocemente, $80 \%$ dos casos de HAS são tratados na atenção primária à saúde ${ }^{(7)}$. Os resultados desta pesquisa revelaram ação não efetiva no controle dos fatores de risco cardiovascular entre os idosos pesquisados. Pode-se afirmar que as atividades centradas somente na distribuição de medicamentos, sem contemplar integralmente a proposta do Programa Hiperdia, não são efetivas.

Os fatores de risco explicam aproximadamente $90 \%$ do risco para IAM na população idosa estudada. Muitos destes fatores são responsáveis por outras patologias, desta forma, o alcoolismo está altamente relacionado a Acidente Vascular Cerebral (AVC), o tabagismo é o principal fator etiológico de câncer de pulmão, a obesidade está estreitamente associada à ocorrência de síndrome metabólica. Neste cenário, o principal desafio para o profissional enfermeiro é desenvolver ações concretas de cuidado integral ao idoso, considerando o conhecimento científico e os avanços tecnológicos disponíveis no cenário brasileiro e mundial ${ }^{(20)}$.

\section{CONCLUSÃO}

Considerando que a maioria dos fatores de risco cardiovasculares são modificáveis e que a principal estratégia para a promoção da saúde e prevenção de agravos existente no Brasil é a Estratégia Saúde da Família, sugere-se que os profissionais de saúde reorientem e direcionem as ações de controle para além da medicalização, contemplando as particularidades dos idosos nas ações de educação em saúde. Na perspectiva de realizar o cuidado integral, devem ser consideradas ações coordenadas entre gestores e equipe interdisciplinar que prestam serviços na atenção básica à saúde, bem como investimento na capacitação dos profissionais a fim de instrumentalizá-los para atuar junto à população idosa.

A pesquisa permitiu constatar que os idosos possuem o hábito de fumar, consumir álcool e alimentos hipercalóricos, associado ao sedentarismo, os quais são considerados fatores de risco para IAM, denotando lacunas em ações efetivas voltadas para o seu controle e prevenção.

Considerando que o enfermeiro é um dos principais agentes da educação para a saúde, cabem os seguintes questionamentos: a falta de atuação efetiva junto à população idosa cadastrada no Programa Hiperdia é decorrente de ações de enfermagem centradas na medicalização? A não utilização das diretrizes propostas pelo Ministério da Saúde está relacionada à falta de conhecimento dos profissionais de saúde ou ao não comprometimento dos mesmos?

\section{REFERÊNCIAS}

1. Organização Mundial de Saúde. Cuidados inovadores para condições crônicas. componentes estruturais de ação relatório mundial. Brasília: Organização Mundial de Saúde; 2002.

2. Ministério da Saúde (BR). Diretrizes e recomendações para o cuidado integral de Doenças Crônicas NãoTransmissíveis. Secretaria de vigilância em saúde, série pacto pela saúde, vol.8, Brasília - DF, 2008.

3. Sistema de Informações sobre mortalidade (SIM). Ministério da Saúde. Mortalidade por Doença Isquêmica do Coração (DIC). Secretaria de vigilância em saúde, Informações de Mortalidade e Saúde, Brasil, 2011.

4. Rochas C, Araújo MP, Volsham A, Carvalho LAF, Ribeiro A, Mesquita ET. Evidência de melhora na qualidade do cuidado assistencial no infarto agudo do miocárdio. Arq. Bras. Cardiol. 2010;94(6):726-9.

5. Melo ECP, Carvalho MS, Travassos C. Distribuição espacial da mortalidade por infarto agudo do miocárdio no Município do Rio de Janeiro, Brasil. Cad. Saúde Pública. 2006,22(6):225-36.

6. Avezum A, Piegas LS, Pereira JCR. São Paulo, SP. Fatores de risco associados com infarto agudo do miocárdio na região metropolitana de São Paulo. Uma região desenvolvida em um país em desenvolvimento. Arq. Bras. Cardiol. 2005; 84(3):206-13.

7. IBGE. Instituto Brasileiro de Geografia e estatística. Indicadores de saúde - HAS e DM. Brasil; 2011. 
8. Ministério da Saúde (BR). A vigilância, o controle e a prevenção das doenças crônicas não transmissíveis. Secretaria de Vigilância em Saúde. Situação e desafios atuais. Brasília; 2005.

9. Sánchez RG, Arribas BN, Arroyo MA, Quirogas SV, García IL, Fernández CS. El Proyecto epicardian: Un estudio de cohortes sobre enfermedades y factores de riesgo cardiovascular em ancianos españoles: consideraciones metodológicas y principales hallazgos demográficos. Rev. Esp. Salud Pública. 2004;78(2):243-55.

10. Pereira JC, Barreto SM, Passos VA. O perfil de saúde cardiovascular dos idosos brasileiros precisa melhorar: estudo de base populacional. Arq. Bras. Cardiol. 2008;91(1):1-10.

11. Lanas F, Avezum A, Bautista LE. Risk factors for acute myocardial infarction in Latin America. American Heart Association. 2007;115:1067-74.

12. Envelhecimento ativo: uma política de saúde. Tradução Suzana Gontijo. Brasília: Organização Pan-Americana da Saúde; 2005.

13. Kaplan HI, Sadock BJ. Tratado de psiquiatria. $3^{\mathrm{a}}$ ed. Porto Alegre: Artes Médicas; 1999.

14. Organização Panamericana de Saúde. CARMEN: iniciativa para prevenção integrada de doenças nãotransmissíveis nas Américas. OMS, Brasília; 2003.

15. Cerecero P, Hernández B, Aguirre D, Valdés R, Huitrón G. Estilos de vida associados al riesgo cardiovascular global em trabajadores universitários del Estado de México. Salud Publica Mex. 2009;51(6):4565-73.

16. Runge MS, Ohman EM. Cardiologia de Netter. Porto Alegre: Artmed; 2006

17. Schwanke, CHA. Atualizações em geriatria e gerontologia. $3^{\mathrm{a}}$ ed. Porto Alegre: EdiPUCRS; 2010.

18. Souza LJ, Gicovate Neto C, Chalita FEB, Reis AFF, Bastos DA, Souto Filho JTD, et al. Prevalência de obesidade e fatores de risco cardiovascular em Campos, Rio de Janeiro. Arq Bras Endocrinol Metab. 2003;47(6):669-76.

19. Ministério da Saúde (BR). Secretaria de vigilância em saúde. Informações de Mortalidade em Saúde. Mortalidade por doença Isquêmica do coração (DIC). Brasília: Ministério da Saúde; 2011.

20. Ministério da Saúde (BR). Secretaria de Vigilância em Saúde.Ações transversais da vigilância em saúde: promoção, integração e análise: gestão 2007-2008/ Ministério da Saúde, Secretaria de Vigilância em Saúde. Brasília: Ministério da Saúde; 2009. 